\title{
Study of the Impact of Introducing Smart Meters in the Spanish Electricity Market
}

\author{
S. Martin ${ }^{1}$ and M. Cabral \\ ${ }^{1}$ Department of Electrical Engineering \\ Escuela Politécnica Superior, Universidad de Málaga \\ Ampliación del Campus de Teatinos Málaga, 29010 Málaga (Spain) \\ Phone number:+0034951 952338, e-mail: smartin@uma.es
}

\begin{abstract}
The impact of hourly consumption information provided by smart meters on the operation of a risk averse retailer in the Spanish electricity market is studied. It is assumed the retailer can manage certain amount of consumers' demand to reduce its operation cost. It can operate in the forward market, day-ahead market and balancing market. Uncertainty in energy price in each market and demand from consumers is taken into account through scenario trees. Conditional Value at Risk is used as a risk measure. Model is posed as a linear two stages stochastic program. The model is applied to real data from the Spanish market.
\end{abstract}

Keywords: Smart metering, retailer, uncertainty, demand side management, risk management.

\section{Notation}

\section{A. Indices and Sets}

$s, S \quad$ Index and set for seasons, $s \in S$.

$h, H \quad$ Index and set for hours, $h \in H$.

$c, C \quad$ Index and set for groups of consumers, $c \in C$.

$k f, K F \quad$ Index and set of forward market scenarios, $k f \in$ $K F$.

$k d, K D \quad$ Index and set for day-ahead market scenarios, $k d \in K D$. Each scenario corresponds to a representative day on an hourly basis (a vector of 24 components).

$k d b, K D B$ Index and set for balancing market scenarios, $k d b \in K D B$.

$k c, K C \quad$ Index and set for consumers scenarios, $k c \in K C$. $\{e s c\} \quad$ Set of all scenarios, $\{e s c\}=\{k f, k d, k d b, k c\}$.

\section{B. Parameters}

$P X F_{s}^{k f}$

Energy price in forward market for season $s$ and scenario $k f$, [EUR/MWh].

$P X P D_{s, h}^{k d} \quad$ Price for energy purchased in day-ahead market in season $s$, hour $h$, and scenario $k d$, [EUR/MWh].

$P X S D_{s, h}^{k d} \quad$ Price of energy sold in day ahead market in season $s$, hour $h$, and scenario $k d$, [EUR/MWh].
$P X P D B_{s, h}^{k d b}$ Price of energy purchased in the balancing market in season $s$, hour $h$, and scenario $k d b$, [EUR/MWh].

$P X S D B_{s, h}^{k d b}$ Price of energy sold in the balancing market in season $s$, hour $h$, and scenario $k d b$, [EUR/MWh].

$\operatorname{Pr} F_{s}^{k f} \quad$ Probability of forward market scenario $k f$ at season $s$.

$\operatorname{Pr} D_{s}^{k d} \quad$ Probability of day-ahead market scenario $k d$ at season $s$.

$\operatorname{Pr} D B_{s}^{k d b} \quad$ Probability of balancing market scenario $k d b$ at season $s$.

$\operatorname{Pr} C_{s}^{k c} \quad$ Probability of customers scenario $k c$ at season

$\operatorname{Pr}_{s}^{\{e s c\}}$

$D E_{s, h, c}^{k c}$

$\delta_{s, c}$

$\lambda$ $s$. Joint probability, $\operatorname{Pr}_{s}^{\{e s c\}}=\operatorname{Pr} F_{s}^{k f} \cdot \operatorname{Pr} D_{s}^{k d}$. $\operatorname{Pr} D B_{s}^{k d b} \cdot \operatorname{Pr} C_{s}^{k c}$.

Energy demand of customer group $c$, at season $s$, hour $h$, and scenario $k c$, [Mwh].

Fraction of demand $D E_{s, h, c}^{k c}$ that can be managed.

Risk aversion, $\lambda \in[0,1], \lambda=0$ means risk neutral, and $\lambda=1$ means a very conservative agent.

$\theta \quad$ Confidence level in the Conditional Value at Risk $\left(\mathrm{CVaR}_{\theta}\right), \theta \in[0,1[$.

\section{Variables}

$x f_{s} \quad$ Energy bought in the forward market, it is a constant value for all the hours in the season $s$, [MW]. $x p d_{s, h} \quad$ Energy bought for the retailer in the day-ahead market to be supplied at hour $h$ in season $s$, [MW]. $x s d_{s, h} \quad$ Energy sold for the retailer in the day-ahead market at hour $h$ in season $s$, [MW].

$x p d b_{s, h}^{k c} \quad$ Energy bought for the retailer in the balancing market to be supplied at hour $h$, season $s$, and demand scenario $k c$, [MW].

$x s d b_{s, h}^{k c} \quad$ Energy sold for the retailer in the balancing market at hour $h$, season $s$, and demand scenario $k c$, 
[MW].

$Q_{s}^{\{e s c\},-}$ Auxiliary variable for CVaR calculation, [EUR].

$d s m a_{s, h, c}^{k c}$ Increase of energy demand for consumer group $c$, at hour $h$ in season $s$, and scenario $k c$ because of demand side management, [MWh].

$d s m r_{s, h, c}^{k c}$ Decrease of energy demand for consumer group $c$, at hour $h$ in season $s$, and scenario $k c$ because of demand side management, [MWh].

$\zeta \quad$ Auxiliary variable for $\mathrm{CVaR}_{\theta}$ calculation, [EUR]. It can be interpreted as the Value at Risk (VaR).

\section{Introduction}

The implementation of smart metering is considered a required first step towards the smart grid deployment. Smart meters are being installed in many countries in Europe, like for instance in Italy, Norway, UK and Spain. How the smart meters could be used in smart grids for demand side management, integration of non-dispatchable distributed renewable generation, management of electric vehicles (including the option of vehicle to grid V2G), and distributed storage has been extensively addressed in the literature.

The focus of this work is on the potential benefits of smart metering in the Spanish Electricity Market. Traditional meters in Spain provide a single value at each time that corresponds to the accumulated energy consumption until that moment. This single value is recorded by the corresponding retailer every month or every two months. That means that consumer and provider have no information on how much energy has been consumed in each hour within the measured period (one or two months). They know just the total energy consumption during a whole month or two months. On the other hand, energy prices in the Spanish electricity market are given on hourly basis.

Smart meters will record energy consumption in periods shorter than one hour, in Spain it will be probably every 15 minutes. Using this information:

- Retailers can assign the proper cost to the energy consumption of each customer, according to the hourly energy market price.

- Consumers can manage their demand to save some money.

- Retailers can propose tariffs and contracts for their customers, so they can reduce their operation cost related to risk coverage. In particular the risks associated with uncertainty in market prices and instantaneous demand from consumers.

We focus on the last point and compute the potential cost saving for a risk averse retailer in the Spanish electricity market. The two main results are the retailer cost saving and the new energy cost for consumers. We assume the cost reduction is transferred to consumers (more competitive prices from the retailer) instead of being a net increase in retailer profit. We do not consider particular tariffs or contracts. We compute an upper bound for what can be done, in terms of demand side management, with the information from the smart meters in the best case (without constraints from particular tariffs or contracts). It is assumed a plausible share of demand that can be managed.

We formulate a model for the optimal operation of a retailer in the Spanish market, it will be explain in detail in Section 2 . The retailer can operate in three markets: Forward market, day-ahead market, and balancing market. It has to deal with uncertainty in market prices and demand from consumers, and can manage certain amount of consumers' demand.

We chose the Spanish system because it offers interesting possibilities because of: (i) High penetration of wind generation, (ii) The installation of smart meters is expected to be completed at the end of 2017, (iii) New regulations oriented to demand side management have been approved in 2015, (iv) Apparently, no cost/benefit analysis for the implementation of smart metering in the Spanish system has been published as indicated in [1], despite the fact that a number of reports about recommendations and studies of alternatives for the operation of smart meters have been published by the Spanish National Energy Commission (CNE).

A relevant question about demand side management, as remarked by Shmuel Oren in [2], is the classical dichotomy between commodity and service (quantity and service) remains, and the main question is not the technical implementation, that has been feasible since the beginning of twenty century. The main question is how to deploy a business model appealing for both retailers and consumers. Here we focus on a simpler question, that is to evaluate the potential cost reduction for a retailer by means of demand side management using the information from smart meters.

Many studies have been reported in the literature on how to use the operational capabilities and the information provided by the smart meters to improve the management of risk averse retailers. For instance, in [3] a methodology based in risk-constrained stochastic programming is proposed to help a retailer make decisions on how much to contract in the forward market and the optimal selling price to offer to its consumers. They use a price-quota function to model that clients can choose other provider if the prices are too high. This model is extended in [4] to consider explicitly the consumer behavior and the competence with other retailers. They propose a bi-level approach consisting of a stochastic problem for a risk averse retailer in the upper level, and an optimization problem for customers in the lower level. The objective function for consumers is to minimize the cost they pay for the energy, and they consider the prices from other retailers (exogenous parameters in the whole problem) and a virtual cost for changing from one retailer to other, this cost is also an exogenous parameter that can be fixed to zero.

A similar model is proposed in [5], the problem is posed as a mixed-integer stochastic programming and solved using decomposition and branch-and-bound. The problem is formulated from the point of view of a retailer, that uses the Conditional Value at Risk (CVaR) [6], [7] as a risk measure. In their approach a retailer manages a portfolio and sets the prices in a Time of Use (TOU) tariff for its consumers. They 
assume fully rational consumers, that are price sensitive and always have a complete reaction to prices. Consumers reaction is modelled through their price elasticity, that is given in an hourly basis (or for a number of hours). A base load profile associated with a base price profile is given for consumers. This load profile is reshaped based on the new prices that are set according to the energy prices the retailer gets from the wholesale market. The consumers option of moving to other retailer is modelled through a market share function that is given by exogenous parameters. They only consider one type of tariff.

Here we pose the problem from the point of view of a risk averse retailer assuming it can manage certain amount of the consumer demand (demand side management), and taking into account its participation in the day-ahead, balancing, and forward markets. The main contributions of this work are to answer the questions that follows for the Spanish system:

1) What is the potential benefit for retailers of the information from smart meters?

2) What is the potential benefit for consumers of the information from smart meters?

The rest of the paper is organized as follows. Methodology, assumptions, and mathematical approach are described in Section 2 . Case study and discussion of results are included in Section 3 . Conclusions in Section 4 close the paper.

\section{Methodology and Mathematical Approach}

To evaluate the potential cost reduction for a risk averse electricity retailer operating in the Spanish system, we use a model posed as a classical two stages stochastic programming. The Conditional Value at Risk is used as risk measure. Uncertainty in electricity market prices and demand from consumers are taken into account through scenario trees, both parameters are input data. The scenario trees are generated based on historical data from the Spanish market. To summarize, the main elements considered in the model are:

- Two type of agents, retailers and three groups of consumers (residential, commercial, and industrial).

- Three markets: Forward market, day-ahead market, and balancing market.

- Simulations in a period of 1 year given by a reduced number of representative days. We consider three days for each season (12 days per year).

- Consumers are considered in an aggregated form.

- Retailer problem is posed as a two stage stochastic programming problem, that takes into account the risk through the CVaR of the cost distribution. The objective function minimizes a weighted average of the expected cost and the CVaR of the cost. The weighting factor is the risk aversion $\lambda \in[0,1]$.

- Demand side management is controlled by the retailer. Here is applied the assumption of "best possible case".

Retailer's strategy consist of managing part of consumer's demand, and to buy and sell electricity in the markets to maximize its profit taking an admissible level of risk. The level of risk and also the bounds for the demand that can be shifted are exogenous parameters.

\section{A. Retailer's decision variables}

The retailer's problem includes the following decisions:

- How much to buy in the forward market (medium-term decision). Retailer cannot sell in the forward market, it can only buy. It can make only a contract for each season, and the power contracted has the same constant value for all the hours in the season.

- How much to buy and sell in the spot market (day-ahead, short-term decision).

- How much to buy and sell in the balancing market (shortterm, almost real time decision).

- Where to shift the amount of consumers' demand it can manage. It can be done in day-ahead and/or real time.

\section{B. Mathematical Approach}

The model is posed as a linear programming problem and solved using algorithm CPLEX in software GAMS. Model equations are listed in what follows:

$$
\min _{\substack{x f_{s}, x p d_{s, h}, x s d_{s, h} \\ x p d b_{s, h}^{k c}, s x d b_{s, h}^{k c} \\ d s m a_{s, h, c}^{k c}, d s m r_{s, h, c}^{k c}}}\left\{(1-\lambda) \cdot \mathbb{E}\left[Q_{s}^{\{e s c\}}\right]+\lambda \cdot C V a R_{\theta}\left[Q_{s}^{\{e s c\}}\right]\right\}
$$

Subject to:

$$
\begin{array}{cc}
x f_{s}+x p d_{s, h}-x s d_{s, h}+x p d b_{s, h}^{k c}-x s d b_{s, h}^{k c} \geq & \\
\sum_{c \in C}\left(D E_{s, h, c}^{k c}+d s m a_{s, h, c}^{k c}-d s m r_{s, h, c}^{k c}\right) & \forall s, h, k c \\
x f_{s} \geq x s d_{s, h}+x s d b_{s, h}^{k c} & \forall s, h, k c \\
\zeta+\sum_{s \in S} Q_{s}^{\{e s c\},-} \geq \sum_{s \in S} Q_{s}^{\{e s c\}} & \forall\{e s c\} \\
Q_{s}^{\{e s c\},-} \geq 0 & \forall s,\{e s c\} \\
\sum_{h \in H} d s m a_{s, h, c}^{k c}=\sum_{h \in H} d s m r_{s, h, c}^{k c} & \forall s, c, k c \\
\sum_{h \in H} d s m a_{s, h, c}^{k c} \leq \delta_{s, c} \cdot \sum_{h \in H} D E_{s, h, c}^{k c} & \forall s, c, k c \\
d s m r_{s, h, c}^{k c} \leq D E_{s, h, c}^{k c} & \\
d s m a_{s, h, c}^{k c} \leq 2 \cdot D E_{s, h, c}^{k c} & \forall s, h, c, k c
\end{array}
$$

Where:

$$
\begin{aligned}
Q_{s}^{\{e s c\}}= & x f_{s} \cdot P X F_{s}^{k f} \cdot 24+\sum_{h \in H}\left(x p d_{s, h} \cdot P X P D_{s, h}^{k d}\right. \\
& -x s d_{s, h} \cdot P X S D_{s, h}^{k d}+x p d b_{s, h}^{k c} \cdot P X P D B_{s, h}^{k d b} \\
& \left.-x s d b_{s, h}^{k c} \cdot P X S D B_{s, h}^{k d b}\right)
\end{aligned}
$$


is the retailer's cost for operating (purchasing and selling) in the markets (day-ahead, balancing, and forward) in season $s$ and scenario $\{e s c\}$.

$$
\mathbb{E}\left[Q_{s}^{\{e s c\}}\right]=\sum_{s \in S} \sum_{\{e s c\}} \operatorname{Pr}_{s}^{\{e s c\}} \cdot Q_{s}^{\{e s c\}}
$$

is the retailer's expected cost in a whole year.

$$
C V a R_{\theta}\left[Q_{s}^{\{e s c\}}\right]=\zeta+\frac{1}{1-\theta} \cdot \sum_{s \in S} \sum_{\{e s c\}} \operatorname{Pr}_{s}^{\{e s c\}} \cdot Q_{s}^{\{e s c\},-}
$$

is the Conditional Value at Risk for a confidence level $\theta$ for the retailer's cost distribution.

Retailer's objective function (1) minimizes a weighted average of the cost expectation and the cost $\mathrm{CVaR}_{\theta}$. The weighting factor is the risk aversion $\lambda \in[0,1]$.

Constraint (2) is a balance equation that states the net energy (purchased minus sold) from the markets must be greater or equal to the net energy consumption at each period of time, taking into account demand side management.

Constraint (3) sets that energy sold by the retailer in the markets can not be greater than the energy it buys from the markets at each period.

Constraints (4) and (5) are auxiliary constraints for $\mathrm{CVaR}_{\theta}$ calculation.

Constraints (6)-(9) model the demand side management mechanism. Constraint (6) sets that total demand remains constant, it does not increase or decrease, it is only shifted. Constraint (7) sets an upper bound, a fraction $\delta_{s, c}$ of the initial demand, for the increase of demand because of the demand side management. Constraint (8) indicates the final demand, after the decrease for demand side management, cannot be negative. Finally constraint (9) states that final demand cannot be greater than twice the initial demand, to avoid creating new demand peaks. We think these assumptions about demand side management are plausible for the Spanish system.

\section{Case Study and Results}

The model described in the previous section is applied to data from the Spanish market, [8]. The data set is quite long, so only a few representative values for each parameter are given. The data input for the model consist of: price of energy purchased from the forward market, Table I, price of energy purchased by the retailer from day-ahead market, Table II, price of energy sold by the retailer in day-ahead market, Table III, price of energy purchased by the retailer in the balancing market, Table IV, price of energy sold by the retailer in the balancing market, Table $\mathrm{V}$, and demand from the three kind of customer groups, Table VI.

For each one of the 5 prices considered we have 4 seasons (s), 3 days for station, and 24 hours (h) for each day, that is for each price $4 \times 3 \times 24=288$ values. For demand from customers we have 3 groups of customers, 3 scenarios (days) for each group, and 24 hours for each day, a total of $3 \times 3 \times 24=216$ values. Representative days are chosen using statistical criteria (mean and deviation) over the set of historical data (a whole year).

Using a risk aversion of $\lambda=0.5$ results for a typical demand shifting pattern in a day in spring season are depicted in Fig. 1. The amount of shifted demand is constrained by shifting bounds and for what is economically profitable. To study the bounds of what is economically profitable we solve the model for the shifting bounds in a range from 0.1 to 0.9 of the total demand. The result for the total cost versus the value of demand that can be shifted are depicted in Fig. 2. The maximum cost saving is around $5 \%$ over the value without demand side management.

The solution does not change when the bounds for reallocating demand are greater or equal to approximately $50 \%$ of total demand. That is because it is not profitable to shift more demand, at least in terms of the objective function in our model, that is in expected value taking into accouton the risk. Thus, even if we have the information from smart meters and are free to shift demand, the expected cost reduction is explicitly bounded by market uncertanties.

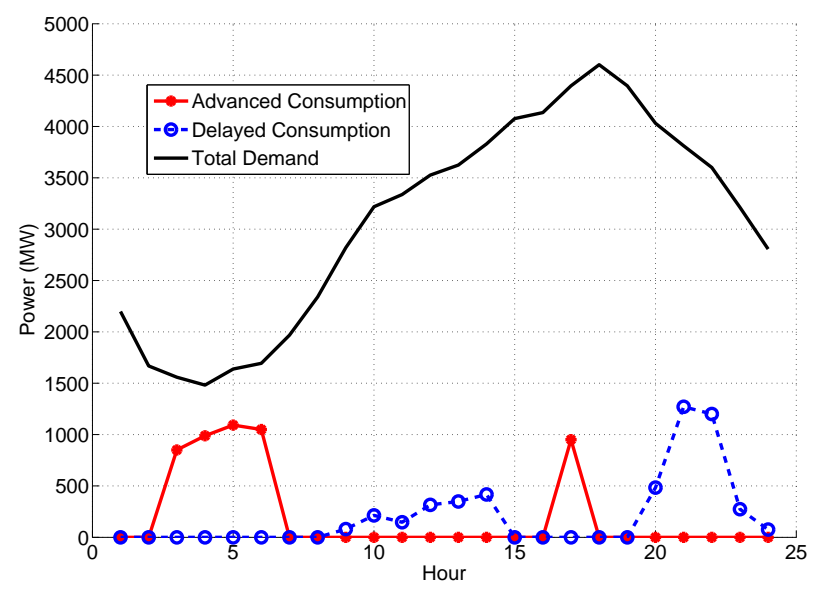

Fig. 1: Demand shifting pattern for a typical day in spring season.

Table I: Scenarios for price (EUR/MWh) of energy purchased in the Spanish forward market in 2015

\begin{tabular}{clll}
\hline & \multicolumn{3}{c}{ Scenario (kf) } \\
\cline { 2 - 4 } (s) & 1 & 2 & 3 \\
1 & 46.22 & 46.24 & 48.94 \\
2 & 51.41 & 51.52 & 54.24 \\
3 & 61.44 & 54.61 & 63.40 \\
4 & 53.95 & 56.58 & 50.88 \\
\hline
\end{tabular}

\section{Conclusions}

To summarize, the main conclusions are:

- The potential benefit for consumers is a relative small fraction of their average cost, around $5 \%$.

- The potential benefit for retailers, even in the case of great flexibility for demand side management, is also a small fraction of their cost. 


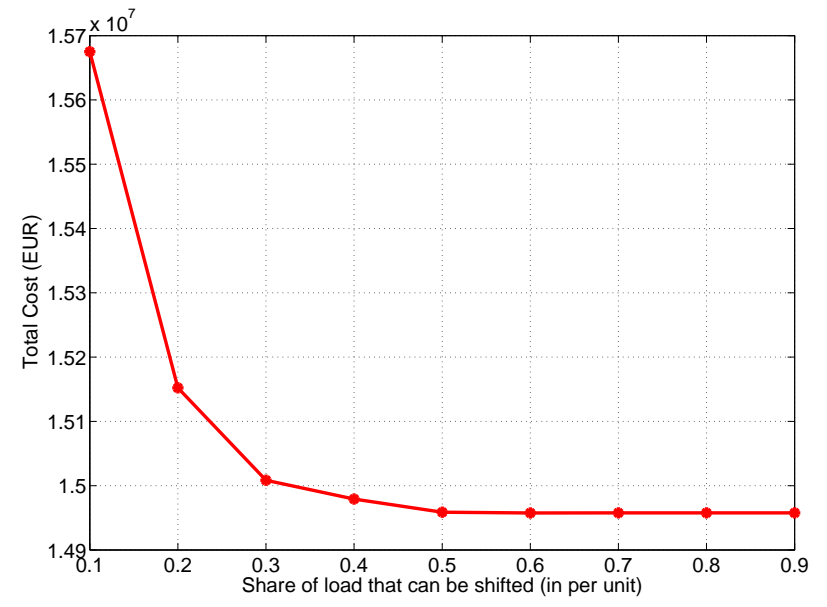

Fig. 2: Total operation cost versus amount of demand that can be shifted.

Table II: Scenarios for price (EUR/MWh) of energy purchased in the Spanish dayahead market in 2015

\begin{tabular}{crrrr}
\hline & & \multicolumn{3}{c}{ Day (kd) } \\
\cline { 3 - 5 }$(\mathbf{s})$ & $(\mathbf{h})$ & 1 & 2 & 3 \\
1 & 1 & 38.24 & 28.99 & 41.69 \\
1 & 12 & 44.58 & 29.76 & 41.83 \\
1 & 24 & 41.79 & 37.19 & 40.94 \\
2 & 1 & 46.49 & 37.89 & 45.49 \\
2 & 12 & 55.93 & 39.84 & 56.04 \\
2 & 24 & 48.71 & 38.45 & 44.37 \\
3 & 1 & 40.02 & 54.43 & 34.54 \\
3 & 12 & 55.99 & 51.73 & 60.54 \\
3 & 24 & 42.05 & 50.23 & 56.44 \\
4 & 1 & 28.97 & 45.74 & 43.46 \\
4 & 12 & 49.35 & 46.14 & 54.87 \\
4 & 24 & 43.72 & 42.46 & 54.32 \\
\hline
\end{tabular}

Table III: Scenarios for price (EUR/MWh) of energy sold in the Spanish day-ahead market in 2015

\begin{tabular}{crrrr}
\hline & & \multicolumn{3}{c}{ Day (kd) } \\
\cline { 3 - 5 }$(\mathbf{s})$ & (h) & 1 & 2 & 3 \\
1 & 1 & 52.01 & 39.43 & 56.71 \\
1 & 12 & 60.64 & 40.48 & 56.90 \\
1 & 24 & 56.84 & 50.59 & 55.69 \\
2 & 1 & 63.23 & 51.53 & 61.88 \\
2 & 12 & 76.08 & 54.19 & 76.22 \\
2 & 24 & 66.26 & 52.30 & 60.35 \\
3 & 1 & 54.44 & 74.03 & 46.98 \\
3 & 12 & 76.15 & 70.36 & 82.34 \\
3 & 24 & 57.19 & 68.32 & 76.77 \\
4 & 1 & 39.40 & 62.22 & 59.11 \\
4 & 12 & 67.12 & 62.76 & 74.63 \\
4 & 24 & 59.46 & 57.75 & 73.88 \\
\hline
\end{tabular}

- In the Spanish system, the main contribution of smart meters, at least in this first step, is to improve the assignation of cost, but not to reduce the cost.

- Increasing the fraction of load that can managed reduces the operation cost for retailers, until reach a certain value above which the operation cost remains constant. That bound depends on market uncertainties.
Table IV: Scenarios for price (EUR/MWh) of energy purchased in the Spanish balancing market in 2015

\begin{tabular}{crrrr}
\hline & & \multicolumn{3}{c}{ Day (kdb) } \\
\cline { 3 - 5 }$(\mathbf{s})$ & $(\mathbf{h})$ & 1 & 2 & 3 \\
1 & 1 & 36.08 & 34.56 & 38.94 \\
1 & 12 & 39.28 & 39.90 & 42.72 \\
1 & 24 & 36.95 & 37.29 & 40.63 \\
2 & 1 & 37.47 & 41.20 & 39.75 \\
2 & 12 & 49.14 & 46.76 & 49.90 \\
2 & 24 & 41.73 & 43.98 & 44.17 \\
3 & 1 & 41.89 & 39.27 & 45.77 \\
3 & 12 & 55.74 & 50.26 & 54.96 \\
3 & 24 & 48.65 & 43.29 & 50.36 \\
4 & 1 & 37.33 & 41.57 & 36.06 \\
4 & 12 & 48.56 & 49.90 & 45.17 \\
4 & 24 & 43.11 & 45.30 & 42.19 \\
\hline
\end{tabular}

Table V: Scenarios for price (EUR/MWh) of energy sold in the Spanish balancing market in 2015

\begin{tabular}{crrrr}
\hline & & \multicolumn{3}{c}{ Day (kdb) } \\
\cline { 3 - 5 }$(\mathbf{s})$ & (h) & 1 & 2 & 3 \\
1 & 1 & 90.20 & 86.39 & 97.34 \\
1 & 12 & 98.19 & 99.76 & 106.80 \\
1 & 24 & 92.38 & 93.23 & 101.57 \\
2 & 1 & 93.68 & 103.00 & 99.37 \\
2 & 12 & 122.86 & 116.91 & 124.74 \\
2 & 24 & 104.33 & 109.94 & 110.43 \\
3 & 1 & 104.73 & 98.18 & 114.42 \\
3 & 12 & 139.35 & 125.65 & 137.39 \\
3 & 24 & 121.63 & 108.22 & 125.91 \\
4 & 1 & 93.32 & 103.94 & 90.15 \\
4 & 12 & 121.40 & 124.75 & 112.92 \\
4 & 24 & 107.77 & 113.25 & 105.48 \\
\hline
\end{tabular}

Table VI: Hourly values for consumers demand $[\mathrm{MWh}]$ in the representative scenarios

\begin{tabular}{crrrr}
\hline & & \multicolumn{3}{c}{ Scenario (kc) } \\
\cline { 3 - 5 }$(\mathbf{c})$ & (h) & 1 & 2 & 3 \\
1 & 1 & 1418.33 & 1174.38 & 1646.69 \\
1 & 12 & 1950.21 & 1659.45 & 2604.06 \\
1 & 24 & 1950.21 & 1557.33 & 2412.59 \\
2 & 1 & 495.13 & 381.25 & 604.55 \\
2 & 12 & 1237.82 & 991.24 & 1503.21 \\
2 & 24 & 606.53 & 479.28 & 702.59 \\
3 & 1 & 285.15 & 223.30 & 343.12 \\
3 & 12 & 338.62 & 269.60 & 400.31 \\
3 & 24 & 249.51 & 198.79 & 285.94 \\
\hline
\end{tabular}

\section{Acknowledgment}

This work was supported in part by Andaluca Tech (International Campus of Excellence), and in part by the Spanish Ministry of Economy and Competitiveness through the project ENE2016-80638-R. The authors would also like to thank the financial support from Junta de Andaluca through Proyecto de Excelencia with Ref. 2011-TIC7070.

\section{REFERENCES}

[1] Javier Leiva, Alfonso Palacios, and José A. Aguado. Smart metering trends, implications and necessities: A policy review. Renewable and Sustainable Energy Reviews, 55:227-233, 2016. 
[2] Shmuel S. Oren and Clay Campaign. A Business Model for Load Control Aggregation to Firm Up Renewable Capacity. Presentation at GDF SUEZ Chair, Energy Economics and Energy Risk Management, 3 July 2014.

[3] M. Carrión, A.J. Conejo, and J.M. Arroyo. Forward Contracting and Selling Price Determination for a Retailer. Power Systems, IEEE Transactions on, 22(4):2105-2114, Nov 2007.

[4] M. Carrión, J.M. Arroyo, and A.J. Conejo. A Bilevel Stochastic Programming Approach for Retailer Futures Market Trading. Power Systems, IEEE Transactions on, 24(3):1446-1456, Aug 2009.

[5] A. Hatami, H. Seifi, and M.K. Sheikh-El-Eslami. A Stochastic-Based
Decision-Making Framework for an Electricity Retailer: Time-of-Use Pricing and Electricity Portfolio Optimization. Power Systems, IEEE Transactions on, 26(4):1808-1816, Nov 2011.

[6] R.T. Rockafellar and S. Uryasev. Optimization of conditional value-atrisk. Journal of Risk, (2):21-41, 2000.

[7] R. Tyrrell Rockafellar and S. Uryasev. Conditional value-at-risk for general loss distributions. Journal of Banking \& Finance, 26(7):14431471, July 2002.

[8] Red Eléctrica de España (REE). Information System of the System Operator. Accessed on 20 October 2016. Available at: www.esios.ree.es. 